\title{
TRATAMIENTO LAPAROSCÓPICO DE LA OBSTRUCCIÓN INTESTINAL POR BRIDAS*
}

\author{
Drs. Nicolás Quezada S. ${ }^{1}$, Felipe León F. ${ }^{1}$, Juan de la Llera K. ${ }^{1}$, \\ Ricardo Funke H. ${ }^{1}$, Mauricio Gabrielli N. ${ }^{1}$, Fernando Crovari E. ${ }^{1}$, \\ Jorge Martínez C. ${ }^{1}$, Camilo Boza W. ${ }^{1}$, Nicolás Jarufe C. ${ }^{1}$ \\ 1 Departamento de Cirugía Digestiva, Escuela de Medicina. Pontificia Universidad Católica de Chile. \\ Santiago, Chile.
}

\begin{abstract}
\section{Laparoscopic treatment of adhesive small bowel obstruction}

Background: Treatment options for adhesive small bowel obstruction (ASBO) involve conservative and surgical management, traditionally through open adhesiolysis. Laparoscopic approach has been performed in recent years; however, limited data exist on its safety and results vary considerably. Our aim is to report our experience of laparoscopic treatment for ASBO. Methods: Retrospective study including patients admitted with the diagnosis of adhesive small bowel obstruction and that were submitted to laparoscopic exploration, between June 2003 and April 2013. We analyzed demographic, surgical variables and outcomes in terms of conversion rate, operative time, re-feeding time and length of stay. Non-parametric tests were used for statistical analysis. Results: Series of 38 patients submitted to laparoscopic exploration, mean age: $51 \pm 16$ years, $47 \%$ male. $53 \%$ had prior intra-abdominal surgeries. Laparoscopic resolution of bowel obstruction was possible in 31 patients $(82 \%)$, with 7 conversions to open surgery. Median operative time was $60(25-180)$ minutes, median re-feeding time was 24 (24-192) hours and median length of stay was 4 (2-52) days. Two patients required re-intervention during their hospital stay, one due to persistent bowel obstruction and one due to ischemic colitis. There were no other complications or mortality. Conclusions: Laparoscopy in adhesive small bowel obstruction was a feasible approach in this series, with good results when laparoscopic resolution is achieved. Patients with no prior surgeries seem to be good candidates for this approach.
\end{abstract}

Key words: Small bowel obstruction, intra-abdominal adherences, laparoscopy.

\section{Resumen}

Introducción: El tratamiento de la Obstrucción Intestinal por Bridas (OIB) incluye alternativas conservadoras y quirúrgicas, esta última tradicionalmente a través de cirugía abierta. El abordaje laparoscópico ha sido incorporado recientemente, sin embargo, existe información limitada sobre su seguridad y sus resultados varían considerablemente. Nuestro objetivo es presentar la experiencia de nuestro centro en el tratamiento laparoscópico de la OIB. Material y Métodos: Estudio retrospectivo incluyendo pacientes con el diagnóstico

*Recibido el 3 de marzo de 2014 y aceptado para publicación el 3 de abril de 2014.

Los autores no declaran conflictos de interés. Sin fuentes de apoyo financiero.

Correspondencia: Dr. Nicolás Jarufe C.

njarufe@med.puc.cl 
de OIB que fueron sometidos a cirugía laparoscópica en nuestro centro, entre junio de 2003 y abril de 2013. Análisis de variables demográficas, quirúrgicas y resultados obtenidos en términos de tasa de conversión, tiempo operatorio, tiempo de realimentación y estadía hospitalaria. Análisis estadístico con pruebas no paramétricas. Resultados: Serie de 38 pacientes, edad promedio: $51 \pm 16$ años, $47 \%$ sexo masculino. 53\% con antecedente de cirugía abdominal previa. Resolución completa por laparoscopía fue posible en 31 pacientes

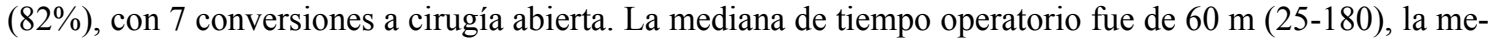
diana de tiempo a la realimentación fue de 24 h (24-192) y la mediana de estadía hospitalaria de 4 (2-52) días. Dos pacientes requirieron reoperaciones durante su hospitalización; uno debido a obstrucción intestinal persistente y otro debido a colitis isquémica. No se presentaron otras complicaciones ni mortalidad en esta serie. Conclusiones: El abordaje laparoscópico en OIB es factible de realizar en pacientes seleccionados, logrando buenos resultados en caso de resolución completa por laparoscopía. Pacientes sin cirugías abdominales previas son buenos candidatos para un abordaje laparoscópico inicial.

Palabras clave: Obstrucción intestinal, adherencias intraabdominales, laparoscopía.

\section{Introducción}

La obstrucción intestinal es uno de los motivos de consulta más frecuentes en los servicios de urgencia quirúrgicos, siendo la causa de hasta un $16 \%$ de los ingresos hospitalarios a los servicios de cirugía ${ }^{1}$.

El intestino delgado es el sitio más frecuente de obstrucción, siendo la patología adherencial o bridas (obstrucción intestinal por bridas, OIB) el factor etiológico más importante de obstrucción intestinal de intestino delgado, dando cuenta del $80 \%$ de las causas de obstrucción intestinal ${ }^{2}$. Otras causas menos frecuentes incluyen la Enfermedad de Crohn, hernias, enfermedad neoplásica y otras ${ }^{3}$.

El escenario clínico es variable dependiendo de la etiología específica, edad, comorbilidades, etc; desde un paciente que requiere sólo descompresión por sonda nasogástrica, hidratación intravenosa y observación clínica hasta casos más extremos que requieren cirugía de urgencia debido a isquemia intestinal y peritonitis secundaria, asociándose a alta letalidad si la intervención quirúrgica es tardía o inadecuadamente realizada.

Se ha estimado que aproximadamente la mitad de los pacientes con OIB requerirán cirugía durante su hospitalización ${ }^{4}$, principalmente debido a sospecha de isquemia intestinal, peritonitis u obstrucción intestinal persistente luego de un período de observación inicial ${ }^{5}$. Tradicionalmente el abordaje quirúrgico para el tratamiento de la OIB ha sido la adherenciolisis a través de cirugía abierta. El año 1991, Bastug publicó la primera adherenciolisis laparoscópica en un paciente con $\mathrm{OIB}^{6}$; desde entonces, la técnica laparoscópica se limitó a escasos reportes debido a la complejidad del procedimiento, escasa experiencia laparoscópica y la alta frecuencia de lesiones inadvertidas de intestino delgado durante el procedimiento. Sin embargo, hoy en día existe un creciente número de publicaciones que muestran resultados prometedores para el abordaje laparoscópico ${ }^{5,7-10}$.
Las potenciales ventajas de este abordaje mínimamente invasivo incluyen menor estadía hospitalaria, menor dolor postoperatorio, menor íleo postoperatorio y menos adherencias intra-abdominales postoperatorias $^{11,12}$. Sin embargo, existe información limitada sobre la seguridad de la laparoscopía en OIB y sus resultados en términos de exactitud diagnóstica y tasas de conversión varían considerablemente entre las distintas series publicadas ${ }^{7-9}$.

En nuestro país no existen hasta la fecha experiencias publicadas de abordaje laparoscópico en esta enfermedad. Por lo anterior, el objetivo de este estudio es presentar la experiencia de nuestro centro en el tratamiento laparoscópico de la OIB.

\section{Material y Método}

Análisis retrospectivo de la base de datos histórica del Departamento de Cirugía Digestiva de la Pontificia Universidad Católica de Chile, entre junio de 2003 y abril de 2013. Se incluyeron a los pacientes hospitalizados con el diagnóstico de OIB y que fueron sometidos a exploración quirúrgica por vía laparoscópica durante su hospitalización.

El diagnóstico de OIB fue realizado en base al cuadro clínico y hallazgos radiológicos. A todos los pacientes incluidos en este estudio se les realizó una tomografía axial computada (TC) de abdomen y pelvis con contraste intravenoso al momento de la admisión, la cual fue concordante con OIB. Pacientes con diagnóstico preoperatorio de obstrucción intestinal de otra etiología como tumores, hernias, bezoares, etc. fueron excluidos del estudio.

Debido a que en nuestra institución no existe un protocolo específico de tratamiento de la OIB, la indicación quirúrgica y el abordaje laparoscópico fueron decididos por el cirujano tratante basado en su juicio clínico, así como en los hallazgos de laboratorio e imagenológicos. A todos los pacientes incluidos en este estudio se les propuso un abordaje laparoscópico y se les explicó la posibilidad de con- 
versión a cirugía abierta, firmando el consentimiento informado de nuestra institución.

Se recolectaron datos demográficos y antecedentes mórbidos como cirugías intra-abdominales previas. Además se registraron variables quirúrgicas como diagnóstico final, tipo de cirugía realizada, técnica utilizada para acceder a la cavidad peritoneal, necesidad de conversión a cirugía abierta, necesidad de resección intestinal, tiempo operatorio, tiempo a la realimentación en el período postoperatorio, estadía hospitalaria, reoperaciones, complicaciones y mortalidad.

El análisis estadístico se realizó en forma descriptiva utilizando pruebas no paramétricas, utilizando el software Statistical Package for the Social Sciences versión 21.0 (SPSS, Chicago, IL, USA).

\section{Resultados}

Durante el período analizado, 282 pacientes fueron sometidos a cirugía debido a OIB en nuestra institución. De éstos, 38 pacientes $(13,4 \%)$ fueron operados por abordaje laparoscópico, siendo analizados en este estudio.

La edad promedio fue de $51 \pm 16$ años (rango, 25-84) y 18 pacientes (47\%) de sexo masculino. Veinte pacientes $(53 \%)$ presentaban el antecedente de cirugía intra-abdominal previa, tres de ellos tenías dos cirugías previas y uno de ellos presentaba tres cirugías previas. De los pacientes sin cirugías intra-abdominales previas (18), dos presentaban el antecedente de reparación herniaria inguinal, sin ingreso a la cavidad peritoneal según lo descrito en los protocolos quirúrgicos (Tabla 1).

La mediana de duración de los síntomas previo a la consulta fue de $48 \mathrm{~h}$ (rango, 6-196). Tal como se mencionó previamente, todos los pacientes fueron estudiados con tomografía axial computada de abdomen y pelvis con contraste intravenoso al momento de la admisión, siendo concordante con OIB. La mediana de tiempo entre el ingreso hospitalario y la indicación quirúrgica fue de 14 h (rango, 0-96), de acuerdo al juicio clínico del cirujano tratante. Los hallazgos de laboratorio e imagenológicos son descritos en la Tabla 2.

La técnica utilizada para acceder a la cavidad peritoneal fue el trocar de Hasson en 17 pacientes $(45 \%)$, trocar de visión directa en 13 pacientes $(34 \%)$ y aguja de Veress en 8 pacientes $(21 \%)$. No se produjeron lesiones intra-abdominales con ninguna de las técnicas utilizadas en esta serie. El número y localización de los trocares laparoscópicos utilizados no fue estandarizado, siendo decidido por la preferencia de cada cirujano durante la cirugía.

La exploración completa y resolución de la obstrucción intestinal por laparoscopía fue posible en
31 de 38 pacientes $(82 \%)$. Siete pacientes requirieron conversión a cirugía abierta (18\%); tres debido a dificultad diagnóstica $(35 / 38=92 \%$ de certeza diagnóstica), dos debido a isquemia intestinal y necesidad de resección intestinal, uno debido a resección de tumor de intestino delgado y uno debido a dificultad técnica durante la liberación de adherencias. El detalle de los diagnósticos postoperatorios se encuentra en la Tabla 3.

Respecto a los resultados quirúrgicos, la mediana de tiempo operatorio fue de $60 \mathrm{~m}$ (rango, 25-180), la mediana de tiempo a la realimentación fue de $24 \mathrm{~h}$ (rango, 24-192) y la mediana de estadía hospitalaria fue de 4,0 días (rango, 2-52).

Dos pacientes fueron reoperados durante su hospitalización; un paciente debido a obstrucción intestinal persistente que requirió una adherenciolisis abierta al quinto día postoperatorio y un paciente que presentó una hemorragia digestiva baja secundaria a colitis isquémica, requiriendo una hemicolectomía izquierda al séptimo día postoperatorio.

No se produjeron lesiones inadvertidas intestinales ni otras complicaciones, así como no existió mortalidad en esta serie. A la fecha, un paciente presentó un nuevo episodio de OIB al cuarto año de la cirugía, el cual fue tratado en forma exitosa con una segunda adherenciolisis laparoscópica.

Tabla 1. Cirugías intra-abdominales previas de pacientes operados por abordaje laparoscópico en obstrucción intestinal por bridas

\begin{tabular}{|lc|}
\hline Tipo de cirugía & $\begin{array}{c}\text { n de pacientes } \\
(\mathbf{n}=\mathbf{3 8})\end{array}$ \\
\hline Ginecológica & 7 \\
Apendicectomía abierta & 3 \\
By-pass gástrico laparoscópico & 3 \\
Colectomía abierta & 2 \\
Colecistectomía laparoscópica & 2 \\
Colecistectomía abierta & 1 \\
By-pass gástrico abierto & 1 \\
$\begin{array}{l}\text { Resección quiste hidatídico hepático } \\
\text { abierta }\end{array}$ & 1 \\
$\begin{array}{l}\text { Nefrectomía radical abierta } \\
\text { Gastrectomía total abierta }\end{array}$ & 1 \\
$\begin{array}{l}\text { Instalación de catéter de diálisis } \\
\text { peritoneal }\end{array}$ & 1 \\
$\begin{array}{l}\text { Sin cirugías intra-abdominales previas } \\
\text { (incluyendo dos hernioplastías } \\
\text { inguinales con técnica de Lichtenstein, } \\
\text { sin ingreso a la cavidad peritoneal) }\end{array}$ & 18 \\
\hline
\end{tabular}


Tabla 2. Características clínicas y de laboratorio de pacientes operados por abordaje laparoscópico en obstrucción intestinal por bridas

\begin{tabular}{|ll|}
\hline Variable & Valor $(\mathbf{n}=\mathbf{3 8})$ \\
Hombre:Mujer & $18: 20$ pacientes \\
Edad, promedio \pm desviación estándar & $51 \pm 16$ años \\
Cirugías intra-abdominales previas (\%) & 20 pacientes $(53 \%)$ \\
Pacientes con comorbilidades (\%) & 24 pacientes $(67 \%)$ \\
Tiempo de síntomas previo a consulta, mediana & $48 \mathrm{~h}(\mathrm{rango}, 6-196)$ \\
Proteína C-Reactiva, mediana & $2,8 \mathrm{mg} / \mathrm{dl}($ (rango, $0,1-29)$ (valor normal $0-10)$ \\
Recuento leucocitos, mediana & $10.100 / \mathrm{uL}($ rango, $5.700-36.300)($ valor normal $3.500-10.000)$ \\
Ácido láctico, mediana & $1,7 \mathrm{mg} / \mathrm{dl}($ rango, $0,1-3,5)($ valor normal $0-2,1)$ \\
\hline
\end{tabular}

Tabla 3. Diagnóstico definitivo de pacientes operados por abordaje laparoscópico en obstrucción intestinal por bridas

\begin{tabular}{|lc|}
\hline Diagnóstico definitivo & $\begin{array}{c}\text { n de pacientes } \\
(\mathbf{n}=\mathbf{3 8})\end{array}$ \\
\hline Adherencia única obstructiva $(\%)$ & $16(42 \%)$ \\
Adherencias múltiples postoperatorias $(\%)$ & $19(50 \%)$ \\
Tumor de intestino delgado (\%) & $1(2,5 \%)$ \\
Vólvulo de intestino delgado (\%) & $1(2,5 \%)$ \\
Enteritis infecciosa $(\%)$ & $1(2,5 \%)$ \\
\hline
\end{tabular}

\section{Discusión}

Alrededor del mundo, la mayoría de los cirujanos considera que el abordaje quirúrgico gold standard en el tratamiento de la obstrucción intestinal es la cirugía abierta a través de una laparotomía ${ }^{13}$. Sin embargo, este concepto lentamente ha ido cambiando a medida que los cirujanos han ido adquiriendo más experiencia en habilidades laparoscópicas avanzadas, tal como ha ocurrido en otras patologías como el cáncer colorrectal ${ }^{7}$.

Existen múltiples ventajas de la laparoscopía al compararla con la cirugía abierta. Éstas incluyen la abolición de la incisión de la laparotomía y su mayor período de recuperación postoperatoria, así como la disminución en la incidencia de hernias incisionales y de las adherencias postoperatorias $^{13,14}$. En el caso de la OIB, la laparoscopía es una herramienta diagnóstica y terapéutica, evitando una laparotomía y sus consecuencias ${ }^{7}$. En aquellos pacientes en los cuales sea prudente y necesario la conversión a cirugía abierta, la laparoscopía permite que la incisión de la laparotomía pueda ser dirigida y limitada al sitio de la obstrucción, evitando así grandes incisiones ${ }^{13}$.

Desde el primer reporte de OIB tratada en forma exitosa por laparoscopía por Bastug en $1991^{5}$, posteriormente han sido publicadas numerosas series e incluso algunas revisiones sistemáticas de la literatura sobre el rol de la laparoscopía en la obstrucción intestinal. Farinella y cols el año $2009^{15}$, encontraron que el tratamiento laparoscópico era posible en el 40 a $88 \%$ de los casos, con una exactitud diagnóstica del 60 a $100 \%$.

Recientemente, $\mathrm{O}^{\prime}$ Connor y cols ${ }^{7}$, revisaron sobre 2.000 casos de obstrucción intestinal operadas por vía laparoscópica, reportando una tasa de conversión a laparotomía media del $29 \%$, principalmente debido a adherencias firmes, necesidad de resección intestinal, dificultad diagnóstica y lesiones iatrogénicas del intestino delgado. En los casos de adherencias únicas como etiología de la obstrucción, en el 73,4\% de los casos se pudo completar el tratamiento por laparoscopía en forma exitosa. Junto con lo anterior, identificaron que las tasas de éxito por vía laparoscópica aumentó de 57\% entre los años 1994 y 2001, a $68 \%$ para los estudios publicados después de 2001, reflejando un aumento de la experiencia laparoscópica entre los equipos quirúrgicos?

En nuestra serie fue posible realizar el diagnóstico etiológico en 35 de 38 pacientes operados $(92 \%)$ y se pudo completar el tratamiento por vía laparoscópica en 31 de 38 pacientes (82\%). Sin embargo, debe notarse que 18 pacientes $(47 \%)$ no tenían cirugías intra-abdominales previas, con una TC de abdomen compatible con obstrucción intestinal secundaria a adherencias. Tal como $\mathrm{O}^{\prime} \mathrm{Connor}^{7} \mathrm{y}$ otros autores sugieren ${ }^{16}$, creemos que estos pacientes son los mejores candidatos para ser abordados por laparoscopía, al menos en forma inicial. En éstos, habitualmente la causa de la obstrucción es una ad- 
herencia única (16 en nuestra serie), transformando la adherenciolisis en un procedimiento de menor complejidad y evitando así una laparotomía.

Respecto al acceso a la cavidad peritoneal para la realización del neumoperitoneo, el año 2011 se publicaron las Bologna Guidelines for Diagnosis and Management of Adhesive Small Bowel Obstruction $^{17}$, en las cuales se plantea que un abordaje laparoscópico puede ser realizado utilizando un acceso peritoneal con técnica abierta como el trocar de Hasson (recomendación grado C), especialmente en pacientes con apendicectomía o histerectomía previa $^{17}$. En nuestra serie, además utilizamos la aguja de Veress y el trocar de visión directa en 8 y 13 pacientes respectivamente, sin haberse producido lesiones asociadas. Nuestra particular preferencia en algunos casos por estas técnicas se explica por nuestra experiencia previa en colecistectomía y cirugía bariátrica. Sin embargo, consideramos que la técnica abierta es el gold standard y debe ser de elección al realizar el abordaje laparoscópico de la obstrucción intestinal, especialmente en pacientes con gran distensión abdominal.

La mortalidad y morbilidad publicada de la cirugía laparoscópica en obstrucción intestinal es de $1,5 \%$ y $14,8 \%$ respectivamente ${ }^{7}$. Otros estudios han reportado mortalidad entre 0 a $4 \%$ y morbilidad entre 19 a $28 \%^{8,15}$. Las lesiones iatrogénicas intestinales es uno de los mayores riesgos de la laparoscopía, siendo la complicación más temida, especialmente si éstas no son reconocidas durante la cirugía. La incidencia de enterotomías iatrogénicas reportada es variable entre las distintas series, desde $6,6 \%{ }^{7}$ hasta incluso $36 \%{ }^{18}$, la mayoría de las veces secundaria a la disección de adherencias firmes, manipulación del intestino distendido o a la inserción de los trocares. En nuestra serie no se produjeron enterotomías iatrogénicas, probablemente debido a que muchos pacientes no tenían cirugías intra-abdominales previas y a que la etiología de la obstrucción intestinal era una adherencia única, facilitando el procedimiento.

Múltiples beneficios, previamente mencionados, se logran al realizar un abordaje laparoscópico exitoso en casos de OIB. Éstos incluyen un menor íleo postoperatorio ${ }^{7,18-20}$ y consecuentemente un menor tiempo a la realimentación. En nuestra institución, los pacientes son realimentados una vez que presentan signos de resolución del íleo postoperatorio habitual, manifestado por ruidos intestinales y expulsión de gases por el ano. En nuestra serie, a las $24 \mathrm{~h}$ de la cirugía la mayoría de los pacientes fueron realimentados, lo cual es más precoz que otras series laparoscópicas en $\mathrm{OIB}^{18,21}$. Otro de los beneficios reportados de la laparoscopía en obstrucción intestinal es la disminución de la estadía hospitalaria ${ }^{18,20-23}$, la cual en nuestra serie fue de 4 días, menor que otras series publicadas ${ }^{18,21}$.

En nuestra serie observamos resultados favorables tras el abordaje laparoscópico en este grupo de pacientes. Sin embargo, existen algunas limitaciones en este estudio que pueden explicar nuestros resultados. En primer lugar, al ser un estudio retrospectivo es susceptible al sesgo de selección propio de este tipo de estudios. Por ejemplo, aquellos pacientes con patologías más complejas o imágenes ominosas podrían haber sido excluidos de un abordaje laparoscópico inicial y por ende, no incluidos en el estudio. En segundo lugar, debido a que no se realizó una comparación con el grupo operado por vía abierta, no podemos afirmar con certeza que el tiempo operatorio, tiempo a la realimentación y estadía hospitalaria fueron menores con el abordaje laparoscópico, pese a los buenos resultados obtenidos. Por último, tal como se mencionó previamente, muchos de los pacientes no tenían cirugías intraabdominales previas y su etiología fue una adherencia única obstructiva, facilitando el diagnóstico y posterior tratamiento laparoscópico, explicando nuestros resultados.

En conclusión, el abordaje laparoscópico en la OIB es factible de realizar en pacientes seleccionados, con bajo tiempo operatorio, estadía hospitalaria y tiempo a la realimentación en caso de éxito. Pacientes sin cirugías abdominales previas son buenos candidatos para un abordaje laparoscópico inicial.

\section{Referencias}

1. Maglinte DD, Heitkamp DE, Howard TJ, Kelvin FM, Lappas JC. Current concepts in imaging of small bowel obstruction. Radiol Clin North Am. 2003;41:263-83.

2. Gutt CN, Oniu T, Schemmer P, Mehrabi A, Büchler MW. Fewer adhesions induced by laparoscopic surgery? Surg Endosc. 2004;18:898-906.

3. Miller G, Boman J, Shrier I, Gordon PH. Etiology of small bowel obstruction. Am J Surg. 2000;180:33-6.

4. Ellis H, Moran BJ, Thompson JN, Parker MC, Wilson MS, Menzies D, et al. Adhesion-related hospital readmissions after abdominal and pelvic surgery: a retrospective cohort study. Lancet 1999;353(9163):1476-80.

5. Wang Q, Hu ZQ, Wang WJ, Zhang J, Wang Y, Ruan CP. Laparoscopic management of recurrent adhesive small-bowel obstruction: Long-term follow-up. Surg Today 2009;39:493-9.

6. Bastug DF, Trammell SW, Boland JP, Mantz EP, Tiley $\mathrm{EH} 3^{\text {rd }}$. Laparoscopic adhesiolysis for small bowel obstruction. Surg Laparosc Endosc. 1991;1:259-62.

7. O'Connor DB, Winter DC. The role of laparoscopy in the management of acute small-bowel obstruction: a review of over 2,000 cases. Surg Endosc. 2012;26:12-7.

8. Li MZ, Lian L, Xiao LB, Wu WH, He YL, Song XM. 
Laparoscopic versus open adhesiolysis in patients with adhesive small bowel obstruction: a systematic review and meta-analysis. Am J Surg. 2012;204:779-86.

9. Cirocchi R, Abraha I, Farinella E, Montedori A, Sciannameo F. Laparoscopic versus open surgery in small bowel obstruction. Cochrane Database Syst Rev. 2010;17:CD007511.

10. Mancini GJ, Petroski GF, Lin WC, Sporn E, Miedema BW, Thaler K. Nationwide impact of laparoscopic lysis of adhesions in the management of intestinal obstruction in the US. J Am Coll Surg. 2008;207:520-6.

11. Tittel A, Treutner KH, Titkova S, Ottinger A, Schumpelick V. Comparison of adhesion reformation after laparoscopic and conventional adhesiolysis in an animal model. Langenbecks Arch Surg. 2001;386:141-5.

12. Tsao KJ, St Peter SD, Valusek PA, Keckler SJ, Sharp $\mathrm{S}$, Holcomb GW 3erd, et al. Adhesive small bowel obstruction after appendectomy in children: comparison between the laparoscopic and open approach. J Pediatr Surg. 2007;42:939-42.

13. Dayton MT, Dempsey DT, Larson GM, Posner AR. New paradigms in the treatment of small bowel obstruction. Curr Probl Surg. 2012;49:642-717.

14. Franklin ME Jr, González JJ Jr, Miter DB, Glass JL, Paulson D. Laparoscopic diagnosis and treatment of intestinal obstruction. Surg Endosc. 2004;18:26-30.

15. Farinella E, Cirocchi R, La Mura F, Morelli U, Cattorini L, Delmonaco P, et al. Feasibility of laparoscopy for small bowel obstruction. World J Emerg Surg. 2009 Jan 19;4:3. doi: 10.1186/1749-7922-4-3.

16. Lujan H, Oren A, Plasencia G, Canelon G, Gómez E,
Hernández-Cano A, et al. Laparoscopic Management as the Initial Treatment of Acute Small Bowel Obstruction. JSLS 2006; 10:466-72.

17. Catena F, Di Saverio S, Kelly MD, Biffl WL, Ansaloni L, Mandalá V, et al. Bologna Guidelines for Diagnosis and Management of Adhesive Small Bowel Obstruction (ASBO): 2010 Evidence-Based Guidelines of the World Society of Emergency Surgery. World J Emerg Surg. 2011;6:5. doi: 10.1186/1749-7922-6-5.

18. Chopra R, McVay C, Phillips E, Khalili TM. Laparoscopic lysis of adhesions. Am Surg. 2003;69:966-8.

19. Mathieu X, Thill V, Simoens CH, Smets D, Ngongang $\mathrm{Ch}$, Debergh N, et al. Laparoscopic management of acute small bowel obstruction: a retrospective study on 156 patients. Hepatogastroenterology 2008;55:522-6.

20. Levard H, Boudet MJ, Msika S, Molkhou JM, Hay JM, Laborde Y, et al. Laparoscopic treatment of acute small bowel obstruction: a multicentre retrospective study. ANZ J Surg. 2001;71:641-6.

21. Zerey M, Sechrist CW, Kercher KW, Sing RF, Matthews BD, Heniford BT. The laparoscopic management of small-bowel obstruction. Am J Surg. 2007;194:882-7.

22. Khaikin M, Schneidereit N, Cera S, Sands D, Efron J, Weiss EG, et al. Laparoscopic vs open surgery for acute adhesive small-bowel obstruction: patients' outcome and cost-effectiveness. Surg Endosc. 2007;21:742-6.

23. Wullstein C, Gross E. Laparoscopic compared with conventional treatment of acute adhesive small bowel obstruction. Br J Surg. 2003;90:1147-51. 